\title{
CARACTERÍSTICAS SOCIALES DEL PEQUEÑO PRODUCTOR DEL CENTRO Y SUDOESTE CHAQUEÑO A FINES DE LOS 90.
}

\author{
MGTER. VIVIANA PÉRTILE \\ vpertile@hum.unne.edu.ar \\ Prof. Adjunta \\ Departamento de Geografía \\ Facultad de Humanidades \\ Universidad Nacional del Nordeste (Argentina) \\ Avenida Las Heras $\mathrm{N}^{\circ} 727-$ \\ 3500 - Resistencia - Chaco - Argentina \\ Teléfono/FAX: $0054-3722-446958$
}

\section{PALABRAS CLAVES}

Pequeños productores - Condiciones de vida- Exclusión.

\section{RESUMEN}

El planteo central de este trabajo hace referencia a las transformaciones económicas y sociales que caracterizaron a la década del ' 80 y su notable impacto en las condiciones de vida de la población de la población, a tal punto que crecientes grupos sociales se vieron excluidos de las posibilidades de atender sus necesidades básicas. Dichas transformaciones también abarcaron a las poblaciones dedicadas al agro y la evidencia más notable fue el deterioro del nivel de vida del pequeño agricultor, como consecuencia de la pérdida de competitividad, a lo que debemos sumar la disminución del empleo de mano de obra contratada por los grandes productores -dada la alta tecnificación- lo cual determina una disminución en los ingresos de las familias dedicadas a la agricultura, sobre parcelas medianas o pequeñas.

\section{KEY WORD:}

Little Farming producers - living conditions - excluded

\section{ABSTRACT:}

The key issue in this paper refers to the social and economic changes that characterised the 1980 s and their great impact on the population living conditions, to souch an extent that emerging social grups were excluded from the possibilities of affording for their basic needs. These changes also included population devoted to farming activities and the most clear Prof. Of this was the impoverishment of living conditions of little farmers as a consequence of the loss of competitivity besides the decrease in employment of workers by big farming producers - due to the use of state of art technology; wich determines a decrease in the incomes of the families devoted to agricultural activities in small or medium sized farms. 


\section{CARACTERÍSTICAS SOCIALES DEL PEQUEÑO PRODUCTOR DEL CENTRO Y SUDOESTE CHAQUEÑO A FINES DE LOS 90.}

\section{1.- Introducción}

Prof. Viviana C. Pertile

La República Argentina, país de alrededor de $2.770 .000 \mathrm{Km}^{2}$ y 37.000 .000 de habitantes, es vista en su conjunto como un territorio de grandes contrastes tanto en su geografía natural como humana.

Su extenso territorio aloja diferentes regiones que responden a su situación, clima, relieve, suelos y recursos naturales. El nivel de vida de su población se presenta con fuertes desniveles debido a las diferencias en los ingresos, educación, situación laboral, acceso a la salud, lo que marca un abismo entre riqueza y pobreza no sólo en la sociedad urbana sino también en la rural. En nuestro caso abordaremos un tema que se inscribe en el ámbito rural de la región chaqueña, en particular la provincia homónima.

En la década pasada Argentina asumió en forma abierta un modelo neoliberal que dejó al mercado y a la apertura comercial como agentes reguladores del crecimiento, lo que en los hechos profundizó la incorporación de nuestro país a la "vorágine" de la globalización de la economía y a sus efectos, haciendo más dependiente y vulnerable nuestro tejido productivo, especialmente el algodonero, que se encuentra en desventajas con el del contexto internacional más adelantado. Con ello han aumentado los problemas, multiplicándose la crisis agraria y de alimentación; se han agudizado la marginación, la competencia entre desiguales, la pobreza rural y urbana, los problemas medioambientales, la migración, y el agravamiento de las condiciones de vida, etc. Dentro de este contexto, los agricultores pierden capacidad de desarrollar sus propias opciones y son absorbidos por las innovaciones tecnológicas y los juegos financieros y de mercado de las empresas transnacionales. Por otra parte, dentro de este modelo internacionalizado, los pequeños y medianos productores son perjudicados por las mega-empresas, cuyo esquema de producción se caracteriza por el alto aporte de capital y tecnología.1

Estas transformaciones económicas y sociales que caracterizaron a la década del '80 tuvieron un notable impacto en las condiciones de vida de la población, a tal punto que crecientes grupos sociales se vieron excluidos de las posibilidades de atender sus necesidades básicas (Carlevari 1996). Dichas transformaciones también abarcaron a las poblaciones dedicadas al agro y la evidencia más notable fue el deterioro del nivel de vida del pequeño agricultor, como consecuencia de la pérdida de competitividad, a lo que debemos sumar la disminución del empleo de mano de obra contratada por los grandes productores - dada la alta tecnificaciónlo cual determina una disminución en los ingresos de las familias dedicadas a la agricultura, sobre parcelas medianas o pequeñas. Estos cambios han sido acompañados por un proceso de concentración de la propiedad agropecuaria. Por un lado, los que poseen capital necesario para los requerimientos de la economía actual, aumentan el tamaño de las explotaciones. Por el otro, en algunos casos, la subdivisión de las parcelas usualmente por el acceso a la herencia, aumentó el número propiedades pequeñas que suelen no alcanzar el tamaño mínimo de una unidad de explotación económica (UEE).

${ }^{1}$ Cf. Gaitán Arciniegas, Jorge y Lacki, Polan (1993). 
Desde el punto de vista de la agricultura global, los pequeños agricultores de nuestra provincia no han podido asumir esta visión e incorporar la idea de una agricultura moderna y eficiente como la mejor alternativa para comenzar a solucionar los problemas nacionales, inclusive los del sector urbano industrial. Por el contrario, la agricultura, actividad que debiera contribuir a impulsar el desarrollo, no está cumpliendo con esta función, fundamentalmente porque ha habido un histórico abandono del sector rural. Los agricultores han estado cautivos de ineficiencias crónicas en adquisición y utilización de insumos y equipos, administración de sus predios, conservación y almacenaje de sus cosechas y comercialización de lo producido. Fueron precisamente estas ineficiencias las que determinaron que la mayoría de los pequeños agricultores generaran una producción de volúmenes reducidos, de mala calidad, con costos unitarios de obtención muy altos y los vendieran a bajos precios. Esta falta de rentabilidad determinó la migración rural, propia de los últimos 20 o 30 años, por la expulsión de los agricultores hacia las periferias urbanas. Sus hijos y nietos en la mayoría de los casos son desempleados, cayendo en muchas ocasiones, estimulados por el consumismo, en la tentación del vicio, la prostitución y la delincuencia. De este modo en lugar de poder aportar riquezas y servicios a la sociedad en el campo, constituyen una carga para las ciudades (Lacki, 1995).

Estos hechos sociales, en parte disimulados por las políticas estatales (Plan trabajar, Jefes de hogar, etc.) para evitar una explosión social, están siendo enfrentados por los propios trabajadores del campo que intentan con sus acciones y políticas contrarrestar esta situación mediante su organización tanto en empresas campesinas, como en cooperativas. Todo ello con la idea de defender sus intereses colectivos, rescatar para sí los procesos productivos que les corresponden y su espacio social, en un contexto cada vez más globalizado.

En el marco de la realidad y de las tendencias anteriormente reseñadas, los pequeños agricultores se encuentran atravesando una crisis profunda: precios no remunerativos, productores altamente endeudados y sin posibilidad de acceso al crédito, lo que se traduce en una resultante social y económica que influye fuertemente sobre el contexto global del Chaco.

\section{2.- El contexto espacial}

La provincia del Chaco presenta una actividad económica primaria que se divide básicamente en dos subsectores: el agrícola y el ganadero. La actividad agropecuaria participa en promedio con el $16 \%$ del producto bruto geográfico (P.B.G). provincial; dentro del mismo la agricultura ocupa el primer lugar con el $65 \%$, seguida por la ganadería con un $24 \%$ y en tercer lugar, con un $6 \%$, la actividad forestal, reducida en los últimos años como consecuencia de la sobreexplotación y disminución de la demanda tanto del mercado interno como del internacional (Pertile, 2003).

La base de la estructura productiva agrícola está dada por el cultivo del algodón, primacía que por décadas caracterizó la economía de la región. Las prácticas culturales en los llamados núcleos algodoneros localizados en territorio formoseño, chaqueño, santiagueño y santafecino, responden a una variada gama de causas entre las que se destacan el factor humano, imprimiendo características particulares a la estructura agraria, y un medio natural cuyas condiciones térmicas y pluviométricas aportan condiciones, al menos adecuadas, para el desarrollo del ciclo vegetativo del mismo.

Dentro de este amplio marco, el espacio geográfico que abarca nuestro estudio está 
circunscripto a dos departamentos de la provincia del Chaco: Independencia y General Belgrano. La elección de ambos responde al importante peso del cultivo algodonero en sus estructuras agrarias y económicas, por corresponder a dos subcuencas de producción diferente, la del Centro y Sudoeste, a los elevados porcentajes de explotaciones con extensión inferior a 100 ha. --45,2\% y 54,9\% respectivamente-- a los que consideramos como pequeños productores ${ }^{2}$. Asociado a esto, se consideran también los altos índices de población rural --52,4\% y 48,7\%-- y de población en zonas rurales con necesidades básicas insatisfechas $--64,3 \%$ y $66,3 \%-{ }^{3}$.

\section{3.- Objetivo, metodología y fuentes. La encuesta}

El objetivo de este trabajo es analizar cómo las nuevas pautas económicas en la actividad agropecuaria han provocado el deterioro de la calidad de vida ${ }^{4}$ del pequeño agricultor en el Chaco.

El análisis de la situación social de los pequeños productores se realizó a partir de la utilización de distintas fuentes de información, como fueron el Censo Nacional de Población de 1991; datos del Ministerio de la Producción de la provincia del Chaco y básicamente las encuestas aplicadas a los pequeños productores. EPPACh-01 (Encuesta a Pequeños Productores Algodoneros del Chaco - 2001).

El formulario de encuesta fue elaborado a partir de los objetivos propuestos en el trabajo y de los cuales se derivaron las variables a utilizar. Básicamente se trató de indagar acerca de la situación socio-económica del pequeño productor del Centro y Sudoeste de la provincia del Chaco. En este caso únicamente haremos referencia a la situación social en relación con la calidad de vida ${ }^{5}$.

\section{1.- Características del cuestionario}

La EEPACh-01 se realizó en base a una muestra de 65 productores de ambos departamentos. Se recabaron datos referidos a: la superficie total, tenencia de la tierra, uso del suelo, producción, mano de obra, nivel tecnológico, condiciones de la vivienda, e información sociodemográfica del productor y su familia, entre otros. En total el cuestionario contiene 20 grandes temas, o variables, divididos en subtemas.

Los ejes temáticos de la encuesta están dirigidos a averiguar la calidad de vida $^{6}$ y la

\footnotetext{
${ }^{2}$ Los técnicos del INTA , especializados en producción agrícola afirman que en la actualidad la explotación algodonera para ser rentable debe tener entre 300 y 500 has., por lo tanto las explotaciones de menos de 100 has. pasaron a ser pequeñas explotaciones, respecto a lo que se consideraba unidad económica agrícola algodonera tres décadas atrás.

${ }^{3}$ Se considera Población con Necesidades Básicas Insatisfechas (NBI) a la que se caracteriza por alguna de las siguientes condiciones: habitan más de tres personas por cuarto; - viven en viviendas precarias o de inquilinato; - faltan en ellas retrete con descarga de agua; tuvieran algún niño en edad escolar (6 a 12 años) que no concurra a la escuela. Este índice surge de los datos del Censo Nacional de Población, la información fue procesada por el Instituto Nacional de Estadística y Censos (INDEC, 1994).

${ }^{4}$ Los indicadores utilizados por diversos autores para definir el concepto de calidad de vida son relativos; se afirma que algunos de esos indicadores son objetivos y otros subjetivos, materiales o espirituales; por lo tanto la mayoría de los analistas se conforman con utilizar los mensurables (Yanes y Liberali, 1986) En otras palabras, las variables objetivas que se utilizan para evaluar la calidad de vida de un grupo determinado de habitantes, deberían estar acompañadas por el componente perceptivo, a los efectos de evaluar, de acuerdo a la opiniones de los habitantes, el nivel de satisfacción de sus necesidades y sus preferencias habitacionales y espaciales (variables subjetivas).

${ }^{5}$ Con relación a la encuesta debemos aclarar que se realizó una pasantía en el Instituto Nacional de Tecnología Agropecuaria, Centro Regional Chaco-Formosa, a los efectos de profundizar conocimientos en la elaboración de Muestreo y del Formulario de Encuesta con su correspondiente codificación.

${ }^{6}$ Por lo general el concepto de calidad de vida se relaciona con la satisfacción de las necesidades del ser humano. Las necesidades son aquellas que tiene el individuo y son de diversa índole y naturaleza. El grado de satisfacción (o no satisfacción) de esas necesidades va a definir los niveles de calidad de vida del grupo humano observado (Bravo y Vera, 1993.)
} 
capacidad productiva del pequeño productor del Sudoeste chaqueño. Estos ejes temáticos fueron operacionalizados ${ }^{7}$ en forma de indicadores y preguntas. Nuestro primer paso fue definir conceptualmente las variables.

Para el análisis de calidad de vida del pequeño agricultor, utilizamos las siguientes variables:

- Datos sociodemográficos: consideramos a la edad del productor, educación de los integrantes del grupo familiar, la salud y el hacinamiento. Si bien a la edad del productor fue incluida en el conjunto de las variables que explican la capacidad productiva, el nivel de análisis para apreciar la calidad de vida es diferente.

- Equipamiento de la vivienda: incluimos los servicios básicos como ser: agua para consumo familiar, tipo de baños, iluminación, combustibles para cocinar, comunicaciones.

- Características físicas de las viviendas: nos interesan, fundamentalmente los materiales con que se construyeron las viviendas, es decir tipo de pisos, paredes, techos, puertas y ventanas.

\section{4.- La situación del sector agrícola en el marco de la globalización}

La globalización económico-financiera ha originado una nueva cultura y una nueva política. Cáceres (1998), sostiene que la cultura de la globalización es la cultura capitalista y la nueva naturaleza del proceso político se define alrededor del poder económico de las compañías transnacionales. En efecto, la globalización demanda la liberalización del sector agrícola a través del debilitamiento o de la eliminación de las estructuras institucionales que fueron creadas como soporte de la agricultura. Un ejemplo práctico de esta liberalización, lo constituye la eliminación de las juntas comercializadoras o reguladoras de la actividad agrícola. Esta transnacionalización de las políticas agrícolas elimina objetivos nacionales para el sector, tales como la seguridad alimentaria, la paridad urbano-rural o el apoyo financiero estatal. La liberalización agrícola aumenta el conflicto entre los productores y los comercializadores de productos agrícolas; los productores no tienen movilidad económica y se debilita su estructura social, en tanto que los comercializadores operan a nivel global y la liberalización les permite el acceso a fuentes globales de abastecimiento.

Bajo este modelo transnacional, la importancia política de los agricultores se reduce en forma dramática, ocupando una posición subordinada a los intereses agro-alimentarios. En este esquema los pequeños y medianos productores son perjudicados por las instituciones de agro-negocios ${ }^{8}$, mientras que los intereses de los grandes productores coinciden con las de estas últimas. Dada la concentración de la comunidad agrícola, las Cooperativas y Federaciones fueron perdiendo importancia en los últimos años. Asimismo, el pequeño productor se halla imposibilitado de usar tecnología e insumos que hagan actualmente rentable su trabajo y no han desarrollado una visión global y coherente para conectarse con el proceso de globalización, perdiendo de este modo, la capacidad de desarrollar sus opciones (Cáceres, 1998).

Apoyándonos en las ideas de Charles Tilly (1991), quien sostiene que si las diferentes sociedades van siguiendo procesos de cambio similares con cierta independencia respecto a los demás, deberíamos entonces construir análisis históricos concretos de los amplios

\footnotetext{
${ }^{7}$ La operacionalización de un concepto implica la búsqueda de indicadores concretos, empíricos, capaces de traducir en términos de variables el planteamiento conceptual involucrado (Bravo y Vera, 1993.)

${ }^{8}$ Los agro-negocios están caracterizados por un esquema de producción integrado, con alto aporte de capital y tecnología.
} 
procesos que caracterizan a nuestra época, es decir análisis que se refieran a tiempos, lugares y personas reales, en un momento definido.

Por lo tanto, para comprender la realidad del Centro y Sudoeste chaqueño, debemos partir de considerar el contexto provincial y regional en el que se encuentra inserto dicho espacio en estudio. Para ello no debemos dejar de mencionar que los procesos que condujeron la configuración de la estructura regional Argentina, dieron como resultado una conformación espacial desigual, de acuerdo al grado de penetración, implantación y difusión del sistema de relaciones económico-sociales capitalista en el espacio nacional. De este modo se originaron áreas diferenciadas, por un lado las más desarrolladas, que concentraron históricamente gran parte de la actividad y potencial económico (área metropolitana y otros polos menores como ser Santa Fe, Córdoba) y por otra parte, el resto del país, con características de marginalidad y en algunos casos de extrema pobreza. En definitiva, podemos afirmar que la realidad de cada espacio en estudio debe ser estudiada abarcando todos los procesos que le dieron origen, le imprimieron sus características peculiares, en un momento determinado y en un espacio definido.

El agro argentino desde fines de la década del '80 se encuentra atravesando procesos de significativa reestructuración como ser: cambio de mercados como resultado de la apertura a la economía mundial, procesos de modernización tecnológica, modificaciones en la función del Estado en términos de redistribución de los excedentes agrarios, etc. Esta nueva situación ha determinado el aumento de requerimientos de capital para poder continuar en la producción (Bidaseca, Gras y Mariotti, 2000).

Asimismo, las transformaciones económicas y sociales que caracterizaron a la década del ' 80 tuvieron un notable impacto en las condiciones de vida de la población, a tal punto que crecientes grupos sociales se vieron excluidos de las posibilidades de atender sus necesidades básicas (Carlevari, 1996). Dichas transformaciones también abarcaron a las poblaciones dedicadas al agro y la evidencia más notable fue el deterioro del nivel de vida del pequeño agricultor, como consecuencia de la pérdida de competitividad, a lo que debemos sumarle la disminución del empleo de mano de obra contratada por los grandes productores dada la alta tecnificación- lo cual determina una disminución en los ingresos de las familias dedicadas a la agricultura, sobre parcelas medianas o pequeñas.

Los pequeños agricultores de nuestra región han estado cautivos de ineficiencias crónicas como adquisición y utilización de insumos y equipos, administración de sus predios, conservación y almacenaje de sus cosechas y comercialización de lo producido. Fueron precisamente estas ineficiencias las que determinaron que la mayoría de los pequeños agricultores generara volúmenes reducidos, de mala calidad, con costos unitarios de obtención muy altos y los vendieran a bajos precios ${ }^{9}$. Esta falta de rentabilidad determinó la gran migración rural, propia de los últimos 20 o 30 años, por la expulsión de los agricultores hacia las periferias urbanas.

Dentro de este contexto, los agricultores pierden capacidad de desarrollar sus opciones y son absorbidos por las innovaciones tecnológicas de las empresas transnacionales; por otra parte dentro de este modelo internacionalizado, los pequeños y medianos productores tienden a ser perjudicados por las mega-empresas, cuyo esquema de producción se caracteriza por el alto aporte de capital y tecnología.

\footnotetext{
${ }^{9}$ Podríamos comparar a estos agricultores con lo que Castells denomina trabajadores genéricos, es decir , aquellos que no han tenido capacidad de reprogramarse, por no incorporar información y conocimiento, mas allá de la capacidad de recibir y ejecutar señales. (Castells,1998.:121)
} 


\section{5.- Características sociales}

\section{1.- Concepto de calidad de vida}

Cuando se intenta medir la calidad de vida no resulta sencillo encontrar los instrumentos adecuados. Las pautas culturales de una sociedad plantean una escala de valores que responde a un determinado modelo; según esos modelos, más o menos generalizados, es que se intenta medir la calidad de vida a partir de indicadores productivos o de ingresos económicos. Generalmente se confunde crecimiento económico con equivalente a mejor calidad de vida. Esto no es correcto, por cuanto no se toma en cuenta la distribución del ingreso. Por otra parte, cada ser tiene necesidades de muy diversa índole, que satisfechas le brindan bienestar en relación al ambiente donde vive. Autores como Maslow, Mallmann, Galtung, entre otros, se refieren a necesidades de tipo material y espiritual (Yanes y Liberali, 1986).

En la operacionalización del concepto de calidad de vida se parte de la definición teórica y se reconocen las dimensiones o componentes de necesidad involucrados. Cada componente está formado a su vez por varios aspectos, los cuales se expresan en términos de satisfactores $^{10}$ o disatisfactores de las necesidades implícitas en el mismo y por último se formulan las variables e indicadores que van a permitir medir cada uno de esos aspectos (de Bravo y Vera, ).

En el caso particular que nos ocupa, consideramos pertinente la utilización de los siguientes indicadores para determinar las características de la calidad de vida. Estos hacen referencia a las condiciones de la vivienda rural que involucra a:

$>$ servicios e infraestructura, dentro de los cuales tomamos en cuenta la procedencia y/o abastecimiento de agua para consumo familiar, las características de los sanitarios, tipo de iluminación, combustible usado para cocinar, equipamiento en comunicaciones, es decir si poseen telefonía fija o celular, radios receptores y televisores;

$>$ calidad de la vivienda, referida específicamente a los materiales con que están construidas las mismas, por ejemplo: tipo de pisos (tierra, madera, cemento, mosaico, etc) , materiales con que están construidas las paredes, los techos, las puertas y ventanas.

Otro grupo de indicadores que utilizamos fue el correspondiente a la salud, la educación y el grado de hacinamiento representados en el primer caso por la cobertura en salud de la población, en el segundo por el nivel de escolarización alcanzado y el tercero por la cantidad de persona por cuarto.

\subsection{La vivienda rural : aproximaciones conceptuales}

Si bien la mayoría de las regiones tienden a un acelerado proceso de urbanización con crecimiento preferentemente de las ciudades de tamaño intermedio, hay un gran segmento de la población que aún vive y seguirá habitando los sectores rurales. En este medio, en gran medida, se aloja la pobreza estructural y su dinámica está siendo afectada negativamente en la mayoría de los casos, por la globalización de la economía y sus impactos en la población. Por otra parte, aún no logran llegar al campo políticas y programas de acción adecuados y

\footnotetext{
${ }^{10}$ son los medios que se utilizan para lograr la satisfacción de una necesidad de alimentación y abrigo que satisfacen la necesidad de subsistencia.
} 
eficaces (Gonzalez Claverán, 1998).

En el análisis de la calidad de vida en áreas rurales intervienen numerosos y complejos componentes, que pueden ser agrupados en: Primero el componente demográfico, que considera a los habitantes, la vivienda y los asentamientos rurales. Todos ellos han decaído notablemente, lo cual implica una pérdida significativa del patrimonio económico, cultural y ecológico. Las causas de este fenómeno son simples, por un lado tenemos la gradual reducción del empleo en zonas rurales que se acentúa conforme se tecnifican las mismas y por otro, la existencia de una mejor calidad de vida en las ciudades. En segundo término se encuentra el componente información. La información existente sobre la vivienda rural y la calidad de vida de los asentamientos rurales, sean concentrados o dispersos, es poca, como también ha sido poco o casi nulo el interés por incidir en el mejoramiento de ese hábitat. Luego, el componente analítico que se refiere a la realización de diagnósticos y pronósticos sobre la situación en el medio rural, detectando las acciones que pudieran considerarse estratégicas y su impacto. Finalmente, Gonzalez Claverán, (1998) incluye otros componentes que hacen referencia a la sustenatabilidad del espacio rural, como ser el modelístico e instrumental..

La vivienda rural y sus características, constituyen uno de los indicadores más utilizados en el estudio de la calidad de vida. Considerada desde el punto de vista geográfico, la vivienda rural obedece a la acción de diferentes factores: los fisioecográficos y los antropogeográficos; por lo tanto, para llegar a comprender las modalidades de los diferentes tipos de vivienda, es necesario realizar un minucioso análisis, sin olvidar que la vivienda rural es un verdadero instrumento de trabajo que cumple una función específica de acuerdo con el género de vida de sus ocupantes (Chiozza y Aparicio, 1961).

Sobre las grandes regiones naturales que presenta la Argentina, la actividad agrícola ha creado una gran variedad de paisajes rurales, cuya fisonomía depende en buena medida del destino que se le ha dado a la tierra y de la antigüedad de la radicación de la población. Cristina Aparicio manifiesta que "entre los distintos elementos que contribuyen a definir el paisaje rural, la vivienda es sin dudas el más significativo". (Chiozza y Aparicio, 1961: 456).

La casa expresa la voluntad de arraigo del hombre en el paisaje y revela la capacidad de sus ocupantes para adaptarse a las condiciones de su ambiente natural, respondiendo a las exigencias de su género de vida, al tipo de tenencia de la tierra, a los recursos económicos de que disponen, las circunstancias históricas, etc.. De este juego de factores es posible encontrar una gran variedad de viviendas rurales. Pese a ello, todas presentan un rasgo común: han sido concebidas para la función rural, que contempla la satisfacción de la necesidad de albergar a los habitantes, proteger sus implementos, sus cosechas y animales (Chiozza y Aparicio, 1961)

Existen diversos criterios para establecer una caracterización de las viviendas rurales. Nosotros empleamos la tipología utilizada por el Censo Nacional de Población y Vivienda $1991^{11}$, referido a los tipos de viviendas, materiales predominantes utilizados para la construcción, provisión de agua, tipo de sanitarios, hacinamiento, entre las características

\footnotetext{
${ }^{11}$ Se define a la vivienda como el recinto construido para alojar a personas. También se considera como viviendas los locales no destinados originalmente a alojar a personas pero que el día del Censo fueron utilizadas con ese fin. Según las definiciones y tipologías utilizadas por el Censo consultado, utiliza varios tipos de viviendas: casa, casa tipo A, casa tipo B, rancho o casilla, entre otros.
} 
más útiles para la evaluación de la calidad de vida. Nuestro espacio rural objeto de estudio, no escapa a las generalidades de las áreas rurales de los países en vías de desarrollo, en tanto se encuentran económicamente deprimidas con relación a los centros urbanos más próximos.

Muchos habitantes del campo no cuentan con ingresos para garantizar un estándard de vida digno, como así tampoco con los recursos deseados para edificar y sostener una vivienda segura desde el punto de vista sanitario. Por ejemplo, en el caso de viviendas rústicas, podría decirse que son potenciales reservorios de diversas enfermedades como el mal de chagas, propio de nuestra región.

Si bien la rusticidad de una vivienda puede ser objetivamente palpable, coincidimos con Rozé y Vaccarezza (1994), cuando manifiestan que muchas veces se habla de la precariedad o rusticidad de una vivienda desde la óptica de las agencias de planificación, definiéndolas de modo arbitrario (en el sentido de su externalidad respecto a las valoraciones sociales de los sujetos involucrados). No es lo mismo decir que ciertas características definidas como negativas, en el concepto de precariedad, tengan las mismas connotaciones en el ámbito urbano que en el rural. Por ejemplo, la electricidad es uno de los componentes más deficitarios, seguramente será valorada como positiva por los pobladores rurales, pero su función en el conjunto habitacional tal vez no sea considerada con la misma urgencia que por un habitante de la ciudad. Otro tanto sucede con los materiales con que se construyen los techos, las paredes, que más allá de las cualidades en términos de higiene, pueden recibir valoraciones diferentes en función de los criterios y acorde al estilo de vida del medio rural.

\section{6.- La situación de los pequeños productores. Resultados de la encuesta}

Con respecto a las condiciones de la vivienda, iniciamos nuestro análisis con los servicios de la misma. En primer lugar analizamos la procedencia del agua para consumo familiar. Advertimos que la mayor proporción de los hogares utilizan el agua proveniente de pozos; en segundo lugar se encuentra el agua almacenada en los aljibes ${ }^{12}$ y por último el agua procedente de bombas y otros; en éste último rubro, en algunos casos se consigna a aguas procedentes de lagunas temporarias.

Figura $n^{\circ} 1$

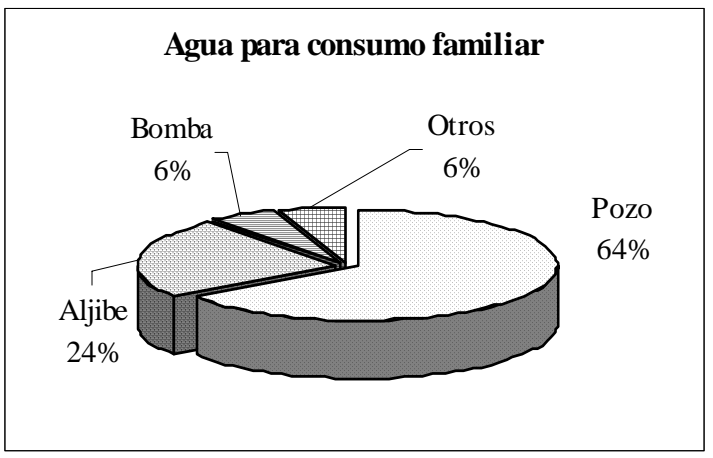

Los resultados arrojados por el indicador correspondiente a tipo de sanitario, muestran datos que si bien no se desconocen, resultan alarmantes. Es sabido que las sociedades rurales tienden a ser conservadoras de sus costumbres, tradiciones y formas de vida, es por ello que no consideran indispensable poseer determinadas condiciones de infraestructura en sus viviendas, como lo es la presencia de un lugar adecuado para el aseo personal. Pese a ello, consideramos importante tener en cuenta el tipo de sanitario utilizado como

\footnotetext{
${ }^{12}$ También conocido como cisterna, donde se almacena agua de lluvia para consumo en épocas de sequías. Generalmente se la destina únicamente para beber, con los riesgos que ello significa, ya que se trata de aguas estancadas, sin ningún tipo de tratamiento o saneamiento. Las familias que consumen este tipo de agua constituyen grupos de riesgo, por cuanto pueden contraer distintas enfermedades.

${ }^{\text {A }}$ EPPCh- $01^{\mathrm{n}}$, : Encuesta a Pequeños Productores del Chaco 2001, ${ }^{\mathbf{n}}$ corresponde a las 65 casos/observaciones.
} 
un indicador relevante de la calidad de vida, por estar íntimamente relacionado con la higiene de las personas y por consiguiente con el estado de la salud las mismas. Del total de viviendas encuestadas, el $84 \%$ contaba solamente con excusado o letrina ${ }^{13}$, pero no disponían de algún tipo de instalaciones adecuadas para el aseo personal, como ser duchas o lavatorios; sólo el $10 \%$ dispone de baño instalado dentro de la vivienda y $6 \%$ restante posee baño instalado fuera de la casa.

Con relación al servicio de alumbrado y combustible usado para cocinar, pudimos advertir que una alta proporción de los encuestados carece de energía eléctrica, siendo la principal fuente de iluminación el gas $(50,8 \%)$ y en segundo lugar el querosenne $(27,7)$ y en menor proporción la corriente eléctrica .

Nos parece pertinente aclarar que si bien los resultados de la muestra indican que el $26,2 \%$ de los encuestados poseen energía eléctrica, no todos la usan, ya que les resulta oneroso

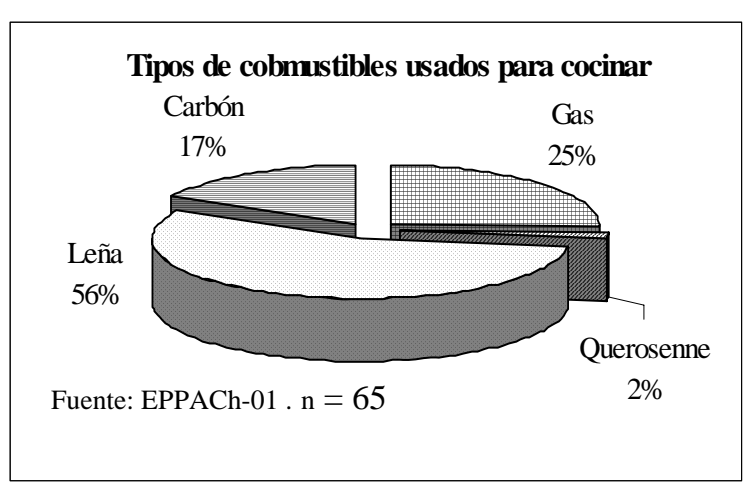
afrontar el pago del servicio. Los agricultores manifestaron que el costo es más elevado que en los centros urbanos próximos, debido a gastos superiores para el mantenimiento del sistema, a la mayor extensión en el tendido de la red de abastecimiento y a la escasez de usuarios. De modo que, pese a la importancia que reviste la electrificación, fundamentalmente por los beneficios que conlleva al sector rural, constituye un servicio muy caro para los pequeños productores.

\section{Figura $n^{\circ} 2$}

En cuanto al combustible utilizado para cocinar, observamos que predomina la leña, usada en fogones, cocinas a leña, hornos, entre los más comunes y representativos de nuestra zona rural.

Dentro de este primer grupo de indicadores incluimos también el equipamiento en comunicaciones.

\section{Cuadro $n^{\circ} 1$}

\begin{tabular}{|c|c|}
\hline Equipamiento en & Comunicaciones \\
\hline Telefonía fija & $1 \%$ \\
\hline Telef. celular & $6 \%$ \\
\hline Radio receptores & $61 \%$ \\
\hline Centro musical & $11 \%$ \\
\hline Televisión & $21 \%$ \\
\hline
\end{tabular}

Éste indicador comprende las comunicaciones con el entorno regional y nacional representadas por la telefonía fija y/o celular y la posibilidad de acceder a la información a través de la radio y televisión. Los datos reflejan un predominio de radio receptores, utilizada en la mayoría de los casos como medio de información y a la vez de distracción; seguido por los televisores-21\%-- y tercer lugar, los centros musicales. Menos significativo fue el porcentaje de productores que poseían teléfonos celulares y fijos.

En relación a la calidad de las viviendas, nuestro observación se centró en los indicadores referidos a los materiales con que están construidas y en averiguar en qué condiciones de

\footnotetext{
${ }^{13}$ Según el censo consultado este tipo de baño corresponde al grupo de retrete sin descarga de agua.
} 
mantenimiento o deterioro se encuentran. Nuestra región, y más específicamente nuestra área de estudio, se encuentra dentro de la zona endémica de enfermedades transmitidas por insectos vectores, tales como la enfermedad de chagas. Asimismo, hay que destacar que otras enfermedades están ligadas con las deficiencias en la vivienda, que incluyen el tipo de materiales usados en los pisos, paredes y techos.

Los indicadores en este caso fueron: el tipo de pisos y materiales con que están construidas las paredes; los techos; las puertas y ventanas. Los pisos son, casi en iguales proporciones de tierra y cemento, dominando ligeramente el primero, que es el menos adecuado para las condiciones sanitarias.

En cuanto al material con que están construidas las paredes de las viviendas, predominan con un $80 \%$ las de ladrillos, en segundo lugar las de barro con $11 \%$, finalmente están las de chapa y otros materiales con el $1 \%$ y $7 \%$ respectivamente.

\section{Cuadro $n^{\circ} 2$}

\begin{tabular}{|l|c|}
\hline \multicolumn{2}{|c|}{ Materiales de Techos } \\
\hline Chapa & $86 \%$ \\
\hline Paja & $3 \%$ \\
\hline Cemento & $2 \%$ \\
\hline Otros & $9 \%$ \\
\hline Puertas y ventanas & \\
\hline Madera & $98 \%$ \\
\hline Chapa & $2 \%$ \\
\hline Otros & $0 \%$ \\
\hline
\end{tabular}

Fuente: EPPACh-01'n= 65
Por último, dentro de este grupo de indicadores, tenemos los materiales utilizados techos y puertas y ventanas; en el caso de los techos, predomina la chapa y la madera es el material con que están hechas las puertas y ventanas.

Otro indicador considerado fue el grado de hacinamiento para lo cual se utilizó como base la definición dada en la publicación Mapas de la pobreza en la Argentina (INDEC,1994) que lo incluye como indicador clave para determinar las necesidades básicas insatisfechas (NBI). El INDEC considera hacinamiento a la presencia de más de tres personas por cuarto.

Según los datos recogidos, predominan las viviendas con dos personas por cuarto y en segundo lugar con 3 personas por cuarto, con lo cual se deduce que no presentan serios problemas de hacinamiento, por cuanto solo el $5 \%$ de los hogares tienen más de tres personas por cuarto.

Las viviendas tienen, por lo general mas de dos cuartos y las familias no son numerosas.

Sintetizando este primer grupo de variables consideramos que las viviendas rurales de ambos departamentos se caracterizan por tener: predominio de pisos de tierra o cemento, paredes exteriores de ladrillo, techos de chapa metálica y puertas y ventanas de madera. Si bien los materiales con que están construidas las viviendas rurales son adecuados para las buenas condiciones sanitarias, con excepción de los pisos de tierra, es preciso destacar que no tienen ningún tipo de mantenimiento ni mejoras con lo cual el estado en que se encuentran las mismas evidencia un alto grado de deterioro.

Con respecto a los servicios, podríamos concluir que hay un elevado grado de carencias, por cuanto la mayor parte de las viviendas no posee energía eléctrica, utilizan combustibles entre los que prácticamente no incluyen el gas; el agua que consumen procede del subsuelo en la mayoría de pozos; tienen retretes sin descarga de agua. 
El tercer grupo de indicadores que utilizamos para evaluar la calidad de vida del pequeño agricultor comprende a: la educación, es decir el nivel de instrucción de los integrantes del grupo familiar y el acceso a la salud representada, en este caso, por la existencia de cobertura en salud, lo cual indicaría de algún modo la posibilidad de atención sanitaria de la población a través del subsistema de seguridad social ${ }^{14}$.

Tal como lo manifestáramos, el proceso de globalización de las economías nacionales provocó un reordenamiento de los sistemas económicos, orientado hacia la construcción de un nuevo orden mundial. Sus efectos se extendieron a todos los ámbitos de la vida política, económica y social de los países.

Los análisis sobre los procesos de desarrollo rural en América Latina, realizados entre otros por Polan Lacky (1995), coinciden en el rol fundamental que juegan la educación, la tecnología y la organización social, donde la educación es considerada como la herramienta principal para alcanzar el desarrollo de un país, y en este caso, el del sector rural. Este planteamiento acerca de la relevancia de la educación ha sido expuesto por numerosos gobernantes latinoamericanos, quienes abogan por una educación de mayor calidad y extensiva a todos los sectores sociales. Sin embargo, las experiencias de educación rural en estos países han estado marcadas por un funcionamiento a espaldas de las realidades socioculturales locales y regionales, y sin tomar en cuenta los problemas, aspiraciones y necesidades de las poblaciones rurales. (Hernández Aracena y Thomas Winter, 1999).

El Informe sobre Desarrollo Humano dado a conocer por el Programa de las Naciones Unidas para el Desarrollo (PNUD) señala que la experiencia parece indicar que aquellas sociedades que invierten por períodos largos en su capital humano, son las que están en condiciones de alcanzar el desarrollo en mejores condiciones y más rápidamente.

En respuesta a lo anterior uno de los indicadores sociales más utilizados para analizar la calidad de vida de una población es la tasa de analfabetismo. Según el informe sobre desarrollo humano en la Argentina, la provincia del Chaco tenía - en 1994- la tasa más alta de analfabetismo con un 12,3\%, en la actualidad ronda alrededor del 10\% (Besil y Piedra, 2000). La educación fue analizada a través del indicador nivel de instrucción formal del jefe de hogar o dueño de la parcela y de todos los integrantes del grupo familiar. Del total de encuestas realizadas (65), obtuvimos una población total de 292 personas, de las

cuales 277 eran mayores de 14 años. De éstas, 160 no completaron el ciclo de educación primaria; 65 sí lo hicieron; 24 tienen incompleto el ciclo secundario y solamente 6 lo completaron.

Finalmente, 21 personas no tienen ningún tipo de educación formal y de ellas, 10 corresponden a los jefes de familia. Se encontró únicamente un individuo con estudios universitarios. Lo más importante en este tema es que más de la mitad de la población no ha completado el nivel primario de la educación y que es importante la proporción de población que se encuentra fuera del sistema educativo, ya sea por no concluir con la enseñanza primaria o por no haber asistido nunca a la escuela.

Otro de los indicadores que utilizamos para evaluar la calidad de vida de los pequeños

\footnotetext{
${ }^{14}$ El Sistema de Servicio de Salud (S.S.S.) se caracterizó históricamente por estar formado por tres subsectores: el público, el privado y el de la seguridad social. 1)subsistema público: los recursos provienen del sistema impositivo; 2)subsistema de seguridad social: financiado por las contribuciones obligatorias a la seguridad social y 3)subsistema privado: financiado con primas de carácter voluntario conforme a los seguros privados de salud. (Fleury, 1997).
} 
productores en el centro y sudoeste chaqueño, fue la cobertura en salud de la población rural, en tanto pueden acceder a atención sanitaria mediante el beneficio de algún tipo de cobertura social, como es el subsistema de Obras Sociales.

El resultado ha sido desalentador por cuanto solamente 16 personas, de las 292 que constituyen el universo poblacional de la muestra, cuentan con cobertura sanitaria a través de la seguridad social, el 5,4\% del total. De ellos, 14 son jubilados - cobertura social a través del PAMI- y 2 personas tienen obra social de los empleados provinciales -INSSEP-. Estos datos dejan a la luz la alta proporción de población que se encuentra excluida del sistema por no poder pagar un servicio de salud prepago -contratación de planes médicos o mutuales- o por no pertenecer al mercado formal de trabajo.

En síntesis, teniendo en cuenta estos dos indicadores, encontramos que más del $50 \%$ del universo poblacional no completó sus estudios primarios y la seguridad social en salud es limitadísima.

\section{7.- Conclusión}

A partir de la información recabada, hemos podido observar que el sentido impuesto por los programas de ajuste a nivel nacional e internacional, lejos de reencauzar el sistema económico de modo progresivo, potenció los problemas del subdesarrollo, creando nuevas y mayores restricciones al crecimiento de los distintos sectores de la provincia --incluido el agro--, ampliando las condiciones de inestabilidad económica.

Salta a la vista que los principios de organización que se pretendieron imponer desde la lógica neoliberal, más que resolver la crisis, aumentaron la posibilidad de que nuestra provincia continúe en estado de crisis. A esto se suma el desmantelamiento de las instituciones del Estado, lo que nos permite hablar de un Estado ausente, ausente de poder regular, justamente cuando la sociedad más lo necesita.

Las condiciones de vida de estas familias de pequeños agricultores, según los resultados de las encuestas, se caracterizan por presentar elementos propios de la pobreza. Los pocos recursos con que cuenta comienzan a mostrar signos de degradación: los suelos están bastante agotados como consecuencia de la práctica del monocultivo algodonero, con las consecuencias lógicas en la producción; disminución de las posibilidades de trabajo como resultado de una reducción en la demanda laboral, especialmente generada por los procesos de mecanización en las explotaciones agrícolas más grandes y más desarrolladas. Esta degradación, más que un problema de insuficiencia de recursos, generalmente es un problema de falta de conocimientos para que los agricultores utilicen racionalmente los recursos que ya poseen y apliquen correctamente tecnologías que sean compatibles con los recursos realmente disponibles.

Utilizamos otras variables, que si bien no las representamos, fueron analizadas e intervienen en la caracterización general del pequeño agricultor. La edad y nivel de educación del productor son variables asociadas; la edad es una característica personal que incide en el comportamiento, incluyendo decisiones relacionadas con el uso de determinadas prácticas agrícolas, uso de tecnología, etc. La edad media de los productores era de 57 años, lo que podría significar una menor permeabilidad a los cambios y a la posibilidad de manejar nuevas prácticas de producción y comercialización. El nivel educativo está asociado a la edad. En un marco general de bajo nivel de educación formal, los productores de más de 40 años de edad, que han pasado su infancia y adolescencia en zonas rurales con limitaciones para el acceso a 
la educación, tienen bajo nivel de escolaridad. Los datos que recogimos nos dieron como resultado que el $73 \%$ no completó la escuela primaria.

En cuanto a la vivienda rural del pequeño productor, podemos concluir diciendo que si bien poseen una vivienda de material, muestran en general un alto grado de deterioro como consecuencia de la falta de mantenimiento por la escasez de medios económicos. Asimismo podríamos decir que se encuentran en estado de abandono, sumidos en la angustia de no saber como hacer para salir de la crisis.

En síntesis podríamos decir que estamos ante la presencia de un proceso de exclusión con relación al sistema económico en su conjunto. Este proceso de exclusión, trae consigo un incremento de la mercantilización donde tanto los insumos como los factores de producción son valorizados según criterios del mercado, logrando en cierta forma la desintegración de las unidades campesinas y su transformación en otros actores sociales agrarios; de manera que no solo se trata de un nuevo modelo productivo agrario, sino que podemos apreciar el surgimiento de un nuevo actor social subordinado.

En definitiva, en los últimos años el sector agrícola local, regional y nacional atravesó y atraviesa profundos e intensos cambios económicos y sociales, que modificaron los escenarios donde se lleva a cabo la producción agrícola.

A modo de cierre decimos que el pequeño agricultor de la provincia del Chaco no está exento de la variedad de cambios que ocurrieron a fines del siglo pasado y en el presente, caracterizados como un conjunto de mutaciones que se sucedieron en las diferentes esferas de nuestra estructura social-económica, cultural, política y social.

\section{Bibliografía y Fuentes}

\section{1. Bibliografía}

1. ARANDA BAEZA, X (1981): Empleo, migración rural y estructura productiva agrícola. Santiago de Chile. FLACSO.

2. ARNAUD, Vicente G. (1999): MERCOSUR. Unión Europea, Nafta, y los procesos de Integración Regional. Buenos Aires. ABELEDO -PERROT.

3. BARBEITO, A y LO VUOLO, R (1995): La Modernización Excluyente. Transformación Económica y Estado de Bienestar en Argentina. Buenos Aires, Losada S.A.

4. BESIL, A. y PIEDRA, D. (2000): "Indicadores sociales relevantes de la región algodonera argentina" En PROINTAL, Programa Integral Algodonero, Capítulo VII. Resistencia, Chaco. Gobierno de la Provincia del Chaco, INTA., UNNE,

5. BIDASECA, K; GRAS, C. y MARIOTTI, D.(2000): "Las transformaciones del mercado de trabajo en el escenario rural: viejos y nuevos procesos de negociación". En: III Congreso Latinoamericano de Sociología del Trabajo. Buenos Aires. Asociación Latinoamericana de Sociología del Trabajo.

6. BRAVO, M. T. D. de y VERA, S. F. de: "Consideraciones metodológicas : una conceptualización del concepto de calidad de vida. En: Revista Geográfica de Venezuela, Volumen 34 . Instituto de Geografía. U.N.L. Mérida. Venezuela

7. BRUNIARD, Enrique D.(1978): El Gran Chaco Argentino. Ensayo de interpretación geográfica, GEOGRÁFICA 4, Resistencia, Chaco. Facultad de Humanidades, UNNE.

8. CÁCERES, Javier (1998): "Globalización y restructuarción del sector agrícola", En II Congreso Técnico Empesarial, Rosario 27 y 28 de agosto de 1998, Federación Agraria Argentina y Programa Fortalecer. 
9. CARDOZIER, U. R (1962): Cultivo y producción del Algodón. México, D.F. Herrero.

10. CARLEVARI, Isidro J. F (1996): La Argentina: Estructura Humana y Económica. $2^{\circ}$ ed. Buenos Aires. Ed. Macchi,

11. CASTELLS, Manuel (1998). "Entender nuestro mundo", en Revista de Occidente, España. Fundación José Ortega y Gasset.

12. CHIOZZA, E y APARICIO, Cristina (1961): La vivienda rural en la Argentina. Suma de Geografía. Buenos Aires. Ed. Peuser

13. CÓRDOBA ORDÓÑEZ, Juan y GARCÍA ALVARADO, José M. (1991): Geografía de la pobreza y la desigualdad. Madrid, España, Ed. Síntesis.

14.DEREWICKI, J: (1997), en Diario Norte, 9 de Abril de 1997. Resistencia, Chaco..

15. DIRY, Jean Paul (1999): "Les espaces ruraux" Paris , Ed. SEDES.

16. Estado del Mundo, 2000, París, Ed. Akal.

17. ESTEFANÍA, Joaquín (1996):La nueva economía. La globalización. Madrid, España. Ed. Debate SA.

18. FANTIN, María Alejandra (1999): Condiciones socioeconómicas y salud en el Gran Resistencia a comienzos de la década de 1990. Colección Tesis de Maestría. Centro de Estudios Avanzados. Córdoba. Argentina. Facultad de Ciencias económicas. U.N.C.

19. FERRER, Aldo (1997): Hechos y ficciones de la globalización. Buenos Aires, Fondo de Cultura Económica, S.A.

20. FLEURY, Sonia, (1997) La implementación del patrón de Seguridad Social en América Latina: cooptación y regulación. En: Estado sin ciudadanos. Buenos Aires. Ed. Lugar.

21. GAITÁN ARCINIEGAS Jorge y LACKI, Polan (1993): "La modernización de la agricultura. Los pequeños también pueden". En Serie Desarrollo Rural No 11. FAO. Organización de las Naciones Unidas para la Agricultura y la Alimentación.

22. GELMAN, Susana (1995): "Análisis del Producto Bruto Geográfico de la Provincia del Chaco. En: Indicadores Económicos. Resistencia, Chaco. Revista del Departamento de Finanzas Año 4, No 22. Facultad de Ciencias Económicas de la UNNE.

23. GIDDENS, Anthony (1999): Un Mundo Desbocado. Los efectos de la globalización en nuestras vidas .Madrid. Ed. Taurus.

24. GIGET, Marc (1995), en GODET, Michel De la anticipación a la acción, Barcelona. Marcumbo editores.

25. GÓMEZ, Maria y Sabeh, Eliana N.(2001) "Calidad de vida. evolución del concepto y su influencia en la investigación y la práctica" http://campus.usal.es/ inico/investigacion/invesinico/calidad.htm\#top.

26. GONZALEZ CLAVERÁN, Jorge (1998): "La vivienda rural y la calidad de vida en los asentamientos rurales en el marco del desarrollo sustentable". Hemeroteca virtual ANUIES, Asociación Nacional de Universidades e Instituciones de Educación Superior. México. http://www.hemerodigital.unam.mx/ANUIES/ipn/academia/15/sec_6.htm.

27. HERNÁNDEZ ARACENA, R y THOMAS WINTER, C (1999): "Educación, Modernidad y Desarrollo Rural" . En : Revista Enfoques Educacionales Vol.2 №1 1999

28. ICAC RECORDER (1997). Información Técnica y Estadística del cultivo del Algodón. En formato Digital (CD-Room).

29. INSTITUTO DE GEOGRAFÍA de la Facultad de Humanidades de la UNNE (1987). Atlas Geográfico de la Provincia Chaco, Tomo I: El Medio Natural. "Geográfica 5" Resistencia, Chaco. 
30. INSTITUTO DE GEOGRAFÍA de la Facultad de Humanidades de la UNNE (1989). Atlas Geográfico de la Provincia Chaco, Tomo II: La tierra y la Agricultura. "Geográfica 6". Resistencia, Chaco.

31. INSTITUTO DE GEOGRAFÍA de la Facultad de Humanidades de la UNNE (1990). Atlas Geográfico de la Provincia Chaco, Tomo III: La Población y el Hábitat "Geográfica 7".: Resistencia, Chaco.

32. INSTITUTO NACIONAL DE TECNOLOGÍA AGROPECUARIA (INTA) EERA de Presidencia Roque Sáenz Peña. Mapa de Capacidad de Uso del Suelo en el Chaco. Escala 1:500.000.

33. INSTITUTO NACIONAL DE ESTADÍSTICAS Y CENSOS (1991): Censo Nacional de Población y Vivienda 1991, Serie B No 25, Total del País, INDEC, Bs,As.

34. INSTITUTO NACIONAL DE ESTADÍSTICAS Y CENSOS (1994): "Mapas de la Pobreza en la Argentina". Comité Ejecutivo para el Estudio de la Pobreza. Bs,As, Argentina.

35. INTA (Instituto Nacional De Tecnología Agropecuaria) (1965): Guías prácticas agrometeorológicas. Publicación micelánea 34, Buenos Aires.

36. INTA (Instituto Nacional de tecnología Agropecuaria). (1967): Plan Nacional de Extensión.

37. INTA (Instituto Nacional de Tecnología Agropecuaria) (1990): Atlas de suelos de la República Argentina, Tomo 1. Capacidad del uso del suelo, Buenos Aires.

38. INTA: Noticias Página Web, Junio 1999.

39. INTA (Instituto Nacional de Tecnología Agropecuaria),UNNE (Universidad Nacional del Nordeste) y Gobierno e la Provincia del Chaco (2000): PROINTAL (Programa Integral Algodonero). Resistencia, Chaco.

40. KLIKSBERG, Bernardo (2000). "Replanteando el problema social: más allá de mitos y slogans", En Hintze, Susana -comp.- Estado y Sociedad, Las políticas sociales en los umbrales del siglo XXI, Buenos Aires. Eudeba.

41. KREBS, Charles J(1985): Ecología. Estudio de la Distribución y de la Abundancia. México, D.F., Harla.

42. KUGLER, Nicolás Diego (1994): Razones para migrar. Una estudio de población del Chaco Algodonero. Tesis de Licenciatura (Inédito). UBA. Buenos Aires.

43. LACKI, Polan, (1995): Buscando soluciones para la crisis del agro, ¿en la ventanilla del banco, o en el pupitre de la escuela?. Santiago de Chile.

44. LARGIÉRE, Robert (1968): El algodón. Técnicas agrícolas y producciones tropicales. Blume. Barcelona.

45. LARRAMENDY, Juan C.(1980): La Economía Algodonera Chaqueña y sus perspectivas. Resistencia, Chaco, Departamento de impresiones y diagramaciones, Cosecha.

46. LOPEZ PIACENTINI, Carlos Primo (1979): Historia de la Provincia del Chaco. Territorio y Provincia. Tomo 5. Región. Resistencia, Chaco.

47. LUMERMAN, Juan Pedro (1998). Crisis social Argentina, Buenos Aires. Lumen.

48. MANOILOFF, Raúl O (2001): El cultivo del algodón desde 1950 a la actualidad (la etapa de crisis) Resistencia, Chaco. Meana y Meana.

49. MINISTERIO DE EDUC, CULTURA, CIENCIA Y TECNOLOGÍA DE LA PROVINCIA DEL CHACO: Ciudades y pueblos de la Provincia del Chaco. Dirección General de Bibliotecas, Biblioteca Pública Leopoldo Herrera Resistencia, 1999.

50. MONETA, Carlos (1994): "El proceso de globalización : Percepciones y desarrollo". En Las Reglas del Juego. América Latina, globalización y regionalismo. Buenos Aires. Ed. Corregidos. 
51. PATRONI, Anibal (1999): "Líneas Básicas para un Programa de Desarrollo Rural Integral". Material suministrado en el cursado de la Maestría en Desarrollo Social, ResistenciaChaco, 2000.

52. PAZ, R. (1997): "Pobreza rural y medio ambiente. Su análisis en un contexto globalizado". En Revista Realidad Económica No 152. Ed. IADE. Buenos Aires

53. PEPE, Norberto C. y DEREWICKI, José V.(1997): Su majestad el Algodón. Resistencia, Chaco, Meana y Meana.

54.PERTILE, Viviana (2003): Ampliación de la frontera agropecuaria chaqueña: El Oeste Chaqueño y el cultivo algodonero. IIGHI, Resistencia, 2003.

55. PROGRAMA ARGENTINO PARA EL DESARROLLO (PAD). Informe Argentino para el Desarrollo, 1995. Honorable Senado de la Nación. Comisión de Ecología y Medio Ambiente. Buenos Aires, 1996.

56. ROCCATAGLIATA, J. A. (1988): La Argentina: geografía general y los marcos regionales. Buenos Aires, Argentina, Grupo Editorial Planeta Argentina.

57. ROFFMAN, A. y MANZANAL, M. (1989). Las economías regionales de la Argentina. Crisis y políticas de desarrollo, Buenos Aires. Bibliotecas Universitarias - Centro Editor de América Latina,

58. ROFMAN. Alejandro B. (2000): Desarrollo Regional y Exclusión Social. Transformaciones y crisis en la Argentina contemporánea. Buenos Aires, Amorrortu editores.

59. SAXE-FERNÁNDEZ, John (1994): Nafta: Los cruces de la geopolítica y geoeconomía del capital. En El Mundo Actual: Situación y Alternativas. México. Centro de Investigaciones Interdisciplinarias en Humanidades.

60. SCHALLER, Enrique C (1986): "La Colonización en el Territorio Nacional del Chaco en el período 1969-1921" En Cuadernos de Geohistoria regional № 12, Resistencia, Chaco. Instituto de Investigaciones Geohistóricas , CONICET-FUNDANORT.

61. SERVICIO METEOROLÓGICO NACIONAL (S/F): Atlas Climático de la República Argentina, período 1921-1950. Buenos Aires. Ed. Fuerza Aérea Argentina

62. SNEDECOR, George (1965): Statical Methods. Applied to Experiments in Agriculture and Biology. Ames, lowa, USA.

63. TILLY, Charles (1991). Grandes Estructuras, Procesos Amplios, Comparaciones Enormes, Madrid. Alianza.

64. VALEIRO, Alejandro (1998): El Nuevo Algodón ¿Son sustentables los cambios recientes del sector algodonero Argentino? Publicación del INTA, Santiago del Estero. En página Web HIPERVÍNCULO httttp://WWW.INTA.com.ar"ww.inta.com.ar

65. YANES, Luis y LIBERALI, Ana María (1986) : Aportes para el estudio del espacio socioeconómico. Buenos aires, Argentina. Ed. El Coloquio.

\subsection{Fuentes}

1. DIRECCIÓN DE CATASTRO DE LA PROVINCIA DEL CHACO : Padrón valuatorio de predios rurales, 1995. Resistencia Chaco.

2. DIRECCIÓN DE ESTADÍSTICAS Y CENSOS: Censo Nacional Agropecuario de 1988. № 17. Provincia del Chaco. Buenos Aires 1991.

3. DIRECCIÓN DE PLANIFICACIÓN, CAPACITACIÓN Y EVALUACIÓN del Ministerio de la Producción de la Provincia del Chaco. Serie Estadística No 16. Enero 1996.

4. INSTITUTO NACIONAL DE ESTADÍSTICAS Y CENSOS: Censo Nacional de Población de 1980. 
Revista Geográfica Digital. IGUNNE. Facultad de Humanidades. UNNE. Año 3- No 5
Enero - Junio 2006. ISSN 1668-5180. Resistencia, Chaco

5. INSTITUTO NACIONAL DE ESTADÍSTICAS Y CENSOS: Censo Nacional de Población de 1991.

6. MINISTERIO DE ECONOMÍA OBRAS Y SERVICIOS PÚBLICOS. .Secretaría de Programación económica. Provincia del Chaco. El Chaco en cifras, Resistencia. Chaco, 1999

7. MINISTERIO DE LA PRODUCCIÓN Provincia del Chaco. Compendio de la Producción, Resistencia. Chaco, 1995

8. MINISTERIO DE LA PRODUCCIÓN Provincia del Chaco. Compendio de la Producción, Resistencia. Chaco, 1998 